\title{
GANGRENE OF THE BLADDER
}

William Britnell ${ }^{1}$, Rachel Hawthorne ${ }^{2}$, Paul Hadway ${ }^{3}$

${ }^{1}$ Specialist Registrar in Urology, Royal Berkshire Hospital, London, UK

${ }^{2}$ Foudation year one doctor in Urology, Royal Berkshire Hospital, London, UK

${ }^{3}$ Consultant Urologist, Royal Berkshire Hospital, London, UK

Corresponding author: williambritnell@gmail.com

Submitted: June 21, 2019. Accepted: July 9, 2019. Published: July 29, 2019.

Case reports of gangrene of the bladder were relatively common in the early twentieth century but there are few reports since the advent of the antibiotic era in the 1940s. 209 cases were reported in $1934^{1}$ with fewer than 30 since. Gangrenous cystitis is recognized as having a high morbidity and mortality rates of up to $35 \%{ }^{2}$ Historically there appeared to be an association with obstructive labour and traumatic delivery but this has been overshadowed by a strong association with diabetes and vascular disease.

\section{CASE HISTORY}

A 75-year-old male presented with septic shock associated with a blocked long-term catheter requiring immediate admission to ITU for inotropic support. He was found on the floor by a relative, the patient reported his catheter had not drained in 2 days. He had a history of type 2 diabetes and dilated cardiomyopathy. He had been a lifelong smoker. He had known benign prostatic enlargement, but had been advised against bladder outlet surgery because of his co-morbidities, hence his long-term catheter for urinary retention. When he was re-catheterised he developed what was assumed to be decompression haematuria and was diagnosed with rhabdomyolysis based on a creatinine kinase of 1500. From blood cultures taken on admission, he grew a fully sensitive Enterobacter cloaca. He remained in intensive care for 48 hours and spent a week on a medical ward.
He was readmitted on three separate occasions with recurrent urinary sepsis and was also seen by the district nurse and $\mathrm{A}+\mathrm{E}$ with recurrent catheter blockages. Urine cultures grew a variety of organisms including enterococcus faecalis, klebsiella pneumoniae, yeasts and vancomycin-resistant Enterococcus faecium. Having had a year of uneventful catheters prior to his ITU admission he was having recurrent catheter blockages requiring replacement almost daily.

He was readmitted under the urology team, a month after his discharge from intensive care, with recurrent catheter blockages. A computed tomography (CT) urogram on admission showed gas in the bladder and bladder wall thickening which was suspicious for malignancy or fistula. Significant efforts were made to exclude a colovesical fistula considering the copious volume of gas seen on the CT. This included a magnetic resonance imaging (MRI) with contrast and a water-soluble contrast enema. Flexible cystoscopy was non-diagnostic due to poor views. The copious purulent exudate was washed out from the bladder via a large bore catheter.

A sagittal CT image with delayed contrast showing gas and debris associated with the anterior bladder wall and a large diameter catheter appropriately sited (Figure 1). The initial report suggested that this represented a bladder tumour or was the consequence of a colo-vesical fistula. 
FIG. 1 A sagittal CT image with delayed contrast showing gas and debris associated with the anterior bladder wall and a large diameter catheter appropriately sited.

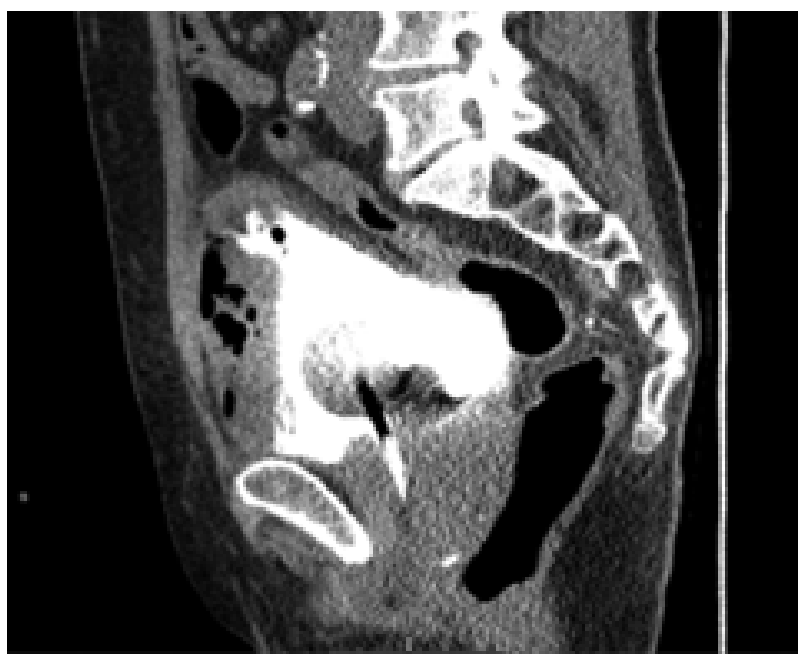

The patient continued to have catheter blockages on the ward so was taken to theatre for a bladder washout. An initial attempt to remove the necrotic tissue was made with the monopolar loop to resect the tissue piecemeal. However, the large volume of fragments of mobile tissue made this technically difficult, so these pieces of mobile tissue were removed whole with the tri-radiate graspers. The final histology report showed necrotic tissues with ghost outlines of deep muscle and fat. The conclusion was this represented prior gangrene of the bladder (Figure 2).

Unfortunately, the patient is not fit enough to have bladder outlet surgery but has not had any further catheter blockages since his final bladder washout in theatre. CT angiogram was performed to assess the feasibility of prostate artery embolization which showed significant vascular disease with stenosis at the origin of the prostate artery. It is likely that this vascular disease has contributed to this pathology. We have arranged for him to have a cystoscopically inserted suprapubic catheter under local anesthetic.

\section{DISCUSSION}

Fulminant cases of gangrene of the bladder, leading to bladder rupture are likely to be picked up during the acute admission. However, cases with a more
FIG. 2 Biopsy of necrotic material from bladder washout and endoscopic debridement showing ghost outlines of deep muscle and fat with patchy mixed inflammation.

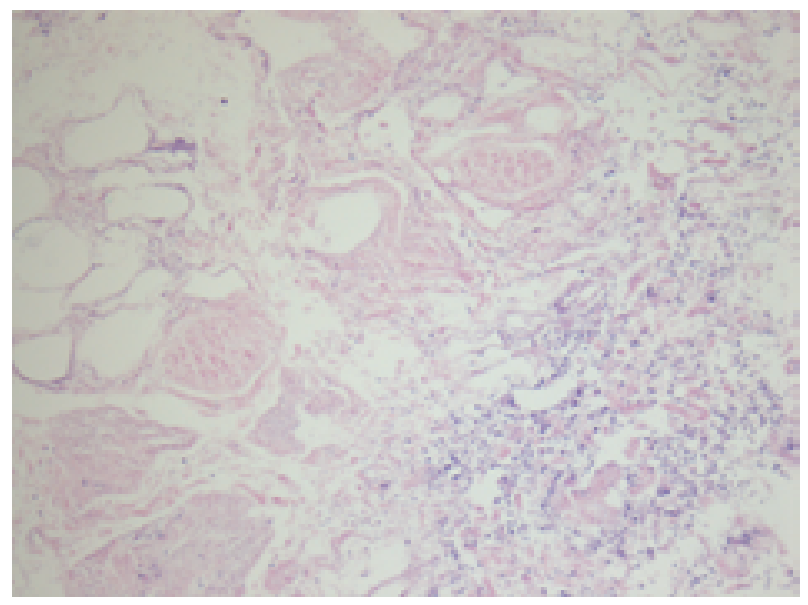

insidious course, without bladder rupture, can lead to a delay in diagnosis and multiple readmissions with the sequelae of gangrenous cystitis, before a diagnosis is made.

It is likely that this patient's initial presentation, necessitating an ITU admission was with gangrenous cystitis and the further admissions were with the sequelae of the necrotic urothelium and detrusor muscle blocking his catheter. There was a delay in making the diagnosis of gangrene of the bladder, but the initial management and supportive care on ITU were appropriate. It is likely that earlier cystoscopic washouts and transurethral debridement would have prevented further catheter blockages and readmissions.

Clinicians should keep gangrene of the bladder on the list of differential diagnoses for recurrent catheter blockages, particularly if recognized risk factors have been present. These include a history of catheterization, vascular disease, diabetes, recent critical illness requiring inotropes and urinary tract infections.

Creatine kinase $(\mathrm{CK})$ released by damaged muscle can be used as an indicator of muscle death. In this case, the markedly elevated CK could have been caused by gangrene of the detrusor muscle, however; it is likely that his presentation following a long lie

J Endolum Endourol Vol 2(3):e25-e27; July 29, 2019.

This article is distributed under the terms of the Creative Commons Attribution-Non

Commercial 4.0 International License. @Britnell et al. 
on the floor was at least partly responsible, with this having caused rhabdomyolysis of skeletal muscle.

We feel the gangrene of his bladder was brought on by his catheter blockage, creating a high-pressure bladder in combination with his poorly controlled diabetes, documented vascular disease of his iliac vessels, and possibly exacerbated by his period of hypotension requiring inotropes in intensive care.

\section{CONCLUSION}

Gangrenous cystitis is a rare presentation, which may not immediately come to the attention of urologists. Fulminant presentations with perforation may lead to diagnosis earlier than cases that do not lead to bladder rupture and urinary peritonitis. The diagnosis should be considered in patients with risk factors and radiological features as described. The diagnosis is confirmed on biopsy, but necrotic tissue present in the bladder may be difficult to manage endoscopically, and open debridement has been reported.

\section{ACKNOWLEDGEMENTS}

With thanks to Dr. Abhisek Ghosh and Dr. Charles Lewis for providing the histopathology slide.

\section{REFERENCES}

1. Stirling WC, Hopkins GA. Gangrene of the bladder. Review of two hundred and seven cases: report of two personal cases. J Urol 1934;31:517. Available at: https://www.auajournals.org/doi/pdf/10.1016/ S0022-5347\%2817\%2972417-2

2. De Rosa A, Amer T, Waraich N, et al. Gangrenous cystitis in a 42-year-old male. Case Rep 2011;2011:bcr1120103526. Available at: https://casereports.bmj.com/content/2011/ bcr.11.2010.3526.full\#ref-1

J Endolum Endourol Vol 2(3):e25-e27; July 29, 2019.

This article is distributed under the terms of the Creative Commons Attribution-Non

Commercial 4.0 International License. CBritnell et al. 